(2) Open Access Full Text Article

\title{
Patency of small laser iridotomy evaluated using anterior-segment optical coherence tomography
}

This article was published in the following Dove Press journal:

Clinical Ophthalmology

2I March 2014

Number of times this article has been viewed

\section{Seigo Nakabayashi \\ Motofumi Kawai \\ Toru Yamaguchi \\ Akitoshi Yoshida}

Department of Ophthalmology, Asahikawa Medical University, Asahikawa, Japan
Correspondence: Motofumi Kawai

Department of Ophthalmology,

Asahikawa Medical University,

Midorigaokahigashi 2-I-I-I,

Asahikawa 078-85I0, Japan

Tel +8I I 66682543

Fax $+8 \mid 166682549$

Email m-kawai@asahikawa-med.ac.jp
Abstract: We report the case of a patient with recurrent acute angle-closure glaucoma who had undergone a previous laser iridotomy. Because the initial iridotomy was small, patency could not be determined by slit-lamp examination. Therefore, anterior-segment optical coherence tomography was used to evaluate the patency. Cross-sectional images showed the presence of a membrane with an anterior bowing configuration at the base of the iridotomy, suggesting that recurrent pupillary block was the causative mechanism. A repeat laser iridotomy was performed, with a resultant decrease in the intraocular pressure and widening of the anterior chamber angle. Anterior-segment optical coherence tomography may be helpful to confirm the status of a laser iridotomy, especially when the iridotomy is small.

Keywords: anterior-segment optical coherence tomography, laser iridotomy, acute angleclosure glaucoma

\section{Introduction}

Anterior-segment optical coherence tomography (AS-OCT) is a noncontact, noninvasive method of imaging the anterior ocular segment. Cross-sectional images of the tissue obtained using this instrument facilitate analysis of the cornea, ${ }^{1}$ anterior chamber depth, iris, and lens. ${ }^{2}$ Trabeculectomy blebs ${ }^{3}$ and scleral flaps ${ }^{4}$ have also been imaged using AS-OCT. In addition, a previous study evaluated laser peripheral iridotomy openings using AS-OCT. ${ }^{5}$ However, to our knowledge, the current report appears to report the first use of AS-OCT to visualize what was likely either an incomplete iridotomy or a membrane over an iridotomy. Although visualization of the anterior lens capsule through an iridotomy opening by slit-lamp examination is necessary to confirm patency, it may be difficult in some patients with a small iridotomy opening.

We report a case of recurrent acute angle-closure glaucoma following a small iridotomy for which patency could not be confirmed by slit-lamp examination. Therefore, to confirm the patency, we used AS-OCT cross-sectional images.

\section{Case report}

A 49-year-old Japanese man presented with blurred vision in both eyes. Intraocular pressure (IOP) was $42 \mathrm{mmHg}$ in the right eye and $70 \mathrm{mmHg}$ in the left eye. Slit-lamp examination showed a cloudy cornea, and gonioscopy showed anterior chamber angle closure in both eyes. The patient was diagnosed with bilateral acute angle-closure glaucoma, and neodymium-doped yttrium aluminum garnet (Nd:YAG) laser iridotomy was performed to eliminate pupillary block in both eyes. After the procedure, IOP decreased to a normal level. 
One month later, however, the patient presented with blurred vision in the right eye. Best-corrected visual acuity was $20 / 16$ bilaterally. IOP was $70 \mathrm{mmHg}$ in the right eye and $23 \mathrm{mmHg}$ in the left eye. Slit-lamp examination of the right eye showed a cloudy cornea. Despite its small size, the previous laser iridotomy was observed at the 10 o'clock position in the iris (Figure 1A). Transillumination through the opening was also observed. Gonioscopy showed angle closure in all four quadrants. Following compression, gonioscopy showed that the closure was partially appositional, and more than half of the angle had synechiae. Based on the sudden IOP elevation associated with a cloudy cornea and blurred vision, we suspected recurrent acute angle-closure glaucoma in this patient, although the initial iridotomy appeared open on slit-lamp examination.

To further evaluate the precise condition of the initial laser iridotomy, we referred to cross-sectional images obtained using AS-OCT (CASIA SS-1000; Tomey Corporation, Nagoya, Japan). The angle mode was used for evaluation. The images showed a membrane that was not observed by slit-lamp examination, with an anterior bowing configuration at the base of the iridotomy (Figure 1B), suggesting increased fluid pressure in the posterior chamber. This observation indicated that pupillary block was the causative mechanism of the increased IOP in our patient, and retreatment with $\mathrm{Nd}$ :YAG laser iridotomy was performed at the same site.

After the procedure, the IOP decreased to $9 \mathrm{mmHg}$. The anterior lens capsule was observed through the opening by
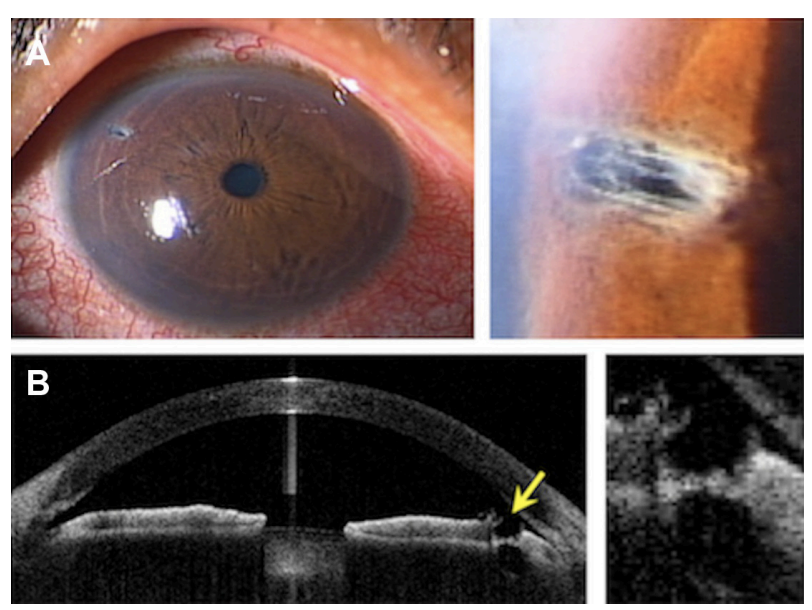

Figure I Before the second laser iridotomy. (A) The initial laser iridotomy appears open, although the opening is small with white collagen strands in the stroma. Myosis is observed due to the preoperative application of topical pilocarpine. (B) A cross-sectional image obtained by anterior-segment optical coherence tomography. A thin membrane with an anterior bowing configuration is seen at the base of the iridotomy (arrow). The intensity of the membrane is not equal to that of the iris stroma. The anterior chamber angle is crowded. Magnified images are also shown. slit-lamp examination (Figure 2A). A cross-sectional image showed a completely penetrated iridotomy with widening of the anterior chamber angle and straightening of the iris (Figure 2B).

\section{Discussion}

In the current case, we believed that recurrent acute angleclosure glaucoma was involved in the pathogenesis, given the sudden IOP elevation associated with a cloudy cornea and blurred vision, although the initial laser iridotomy appeared open based on slit-lamp examination. Indeed, gonioscopic examination showed angle closure in all four quadrants. However, we speculated that occlusion of the initial iridotomy occurred at some level of the iridotomy hole that was not directly observable during slit-lamp examination due to the small opening. We performed AS-OCT to obtain more information about the condition of the iridotomy.

The cross-sectional images clearly showed a membrane with an anterior bowing configuration at the base of the iridotomy, suggesting increased fluid pressure in the posterior chamber due to a functional block between the lens and iris. This observation convinced us to perform another laser iridotomy. Postoperatively, widening of the anterior chamber angle and straightening of the iris occurred and
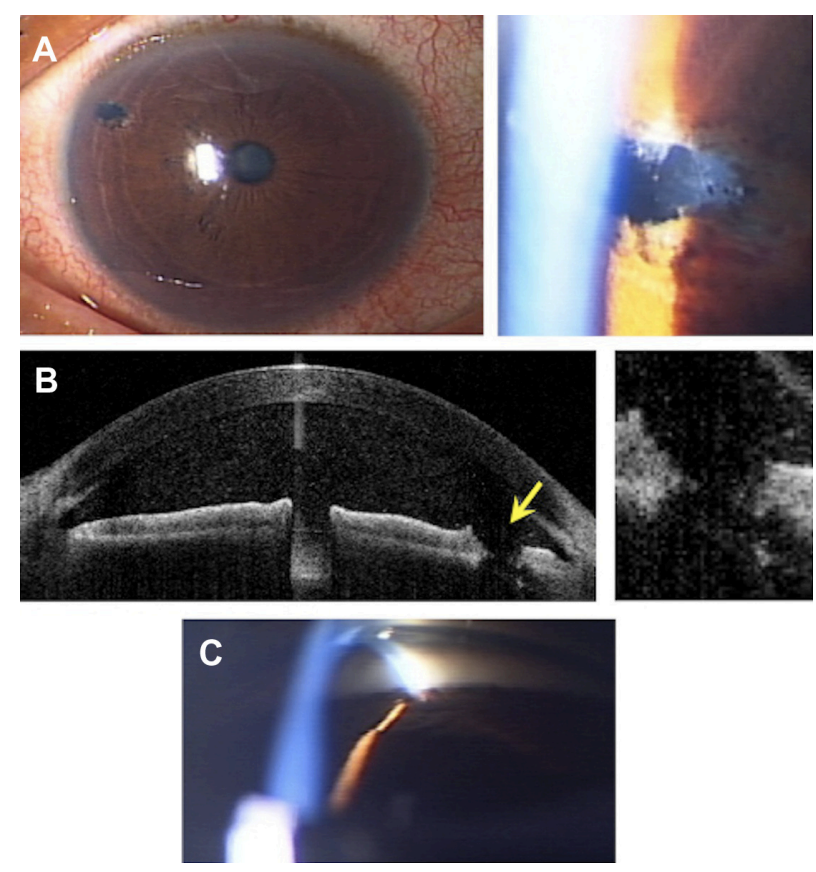

Figure 2 After the second laser iridotomy. (A) The iridotomy is completely penetrated. The anterior lens capsule is seen through the opening. (B) Crosssectional images obtained by anterior-segment optical coherence tomography. The iridotomy is completely penetrated (arrow). Widening of the anterior chamber angle and straightening of the iris are also seen. (C) The plateau iris configuration is seen by gonioscopy. Magnified images are also shown. 
IOP decreased. In this case, we thought that the plateau iris configuration might have contributed partially to the pathogenesis (Figure 2C). Additional laser peripheral iridoplasty will stabilize the IOP over a long period.

Considering that a minimal diameter of 150-200 microns is recommended when performing a laser iridotomy, ${ }^{6}$ the size of the initial iridotomy in our patient was relatively small. Closure of a laser iridotomy can occur either immediately or later after the procedure. Accumulation of pigment granules and debris primarily causes immediate closure. However, localized pigment proliferation causes late closure and leads to development of a transparent, thin, fibrous membrane occluding the opening and regeneration of the iris pigment epithelium from the margins of the iridotomy. ${ }^{7}$ In the current case, the latter mechanism was likely, given that a membrane was clearly present at the bottom of the iridotomy. Because the intensity of the membrane was not equivalent to that of the iris stroma, we speculated that the membrane had newly developed after the initial laser iridotomy. Although late closure is rare with Nd:YAG laser iridotomies compared with argon laser iridotomy, ${ }^{8}$ in our patient the iridotomy closed soon after the initial procedure, despite use of an Nd:YAG laser. We think that the fibrous membrane developed and regenerated at the base of the iridotomy because of an accelerated wound-healing process in our patient, who was relatively young compared with elderly patients with angle-closure glaucoma, who generally require laser iridotomy.

In the current case, an invasive surgery (ie, goniosynechialysis with or without phacoemulsification) was avoided by carefully considering the pathogenesis of the IOP elevation and evaluating the condition of the iridotomy using AS-OCT. If the iridotomy was found to be open only by slit-lamp examination, our young patient might have had to undergo that invasive surgery.
Although visualizing the anterior lens capsule through the iridotomy opening by slit-lamp examination is necessary to confirm the patency, it may be difficult for some patients with a small iridotomy opening. In addition, transillumination can be misleading regarding patency. Therefore, cross-sectional images obtained using AS-OCT might be helpful to confirm the precise condition of iridotomy in those patients.

\section{Disclosure}

The authors have no proprietary interest in any aspect of this report.

\section{References}

1. Chan JB, Yuen LH, Huang EH, et al. Reproducibility of cornea measurements in anterior segment OCT images of normal eyes and eyes with bullous keratopathy analyzed with the Zhongshan Assessment Program. Invest Ophthalmol Vis Sci. 2011;52(12):8884-8890.

2. Lee KS, Sung KR, Shon K, et al. Longitudinal changes in anterior segment parameters after laser peripheral iridotomy assessed by anterior segment optical coherence tomography. Invest Ophthalmol Vis Sci. 2013;54(5):3166-3170.

3. Nakano N, Hangai M, Nakanishi H, et al. Early trabeculectomy bleb walls on anterior-segment optical coherence tomography. Graefes Arch Clin Exp Ophthalmol. 2010;248(8):1173-1182.

4. Inoue T, Matsumura R, Kuroda U, et al. Precise identification of filtration openings on the scleral flap by three-dimensional anterior segment optical coherence tomography. Invest Ophthalmol Vis Sci. 2012;53(13):8288-8294.

5. Chalita MR, LiY, Smith S, et al. High-speed optical coherence tomography of laser iridotomy. Am J Ophthalmol. 2005;140(6):1133-1136.

6. Fleck BW. How large must an iridotomy be? $\mathrm{Br} J$ Ophthalmol. 1990;74(10):583-588.

7. Rich R, Liebmann JM. Laser iridotomy and peripheral iridoplasty. In: Ritch R, Shields MB, Krupin T, editors. The Glaucomas. St Louis, MO: Mosby; 1996:1549-1573.

8. Del Priore LV, Robin AL, Pollack IP. Neodymium: YAG and argon laser iridotomy. Long-term follow-up in a prospective, randomized clinical trial. Ophthalmology. 1988;95(9):1207-1211.
Clinical Ophthalmology

\section{Publish your work in this journal}

Clinical Ophthalmology is an international, peer-reviewed journal covering all subspecialties within ophthalmology. Key topics include: Optometry; Visual science; Pharmacology and drug therapy in eye diseases; Basic Sciences; Primary and Secondary eye care; Patient Safety and Quality of Care Improvements. This journal is indexed on

\section{Dovepress}

PubMed Central and CAS, and is the official journal of The Society of Clinical Ophthalmology (SCO). The manuscript management system is completely online and includes a very quick and fair peer-review system, which is all easy to use. Visit http://www.dovepress.com/ testimonials.php to read real quotes from published authors. 